\title{
Multimodality Imaging of Myocardial Injury and Remodeling
}

\author{
Christopher M. Kramer ${ }^{1}$, Albert J. Sinusas ${ }^{2}$, David E. Sosnovik ${ }^{3}$, Brent A. French ${ }^{1}$, and Frank M. Bengel ${ }^{4}$
}

${ }^{1}$ Departments of Medicine, Radiology, and Biomedical Engineering and the Cardiovascular Imaging Center, University of Virginia, Charlottesville, Virginia; ${ }^{2}$ Cardiovascular Division, Department of Medicine, Yale School of Medicine, New Haven, Connecticut; ${ }^{3}$ Center for Molecular Imaging Research, Massachusetts General Hospital, Boston, Massachusetts; and ${ }^{4}$ Division of Nuclear Medicine, Department of Radiology, Johns Hopkins School of Medicine, Baltimore, Maryland

\begin{abstract}
Advances in cardiovascular molecular imaging have come at a rapid pace over the last several years. Multiple approaches have been taken to better understand the structural, molecular, and cellular events that underlie the progression from myocardial injury to myocardial infarction (MI) and, ultimately, to congestive heart failure. Multimodality molecular imaging including SPECT, PET, cardiac MRI, and optical approaches is offering new insights into the pathophysiology of $\mathrm{MI}$ and left ventricular remodeling in small-animal models. Targets that are being probed include, among others, angiotensin receptors, matrix metalloproteinases, integrins, apoptosis, macrophages, and sympathetic innervation. It is only a matter of time before these advances are applied in the clinical setting to improve post-MI prognostication and identify appropriate therapies in patients to prevent the onset of congestive heart failure.
\end{abstract}

Key Words: MRI; PET; SPECT; myocardial infarction; noninvasive imaging; remodeling

J Nucl Med 2010; 51:107S-121S

DOI: 10.2967/jnumed.109.068221

\section{A} dvances in cardiovascular molecular imaging have come at a rapid pace over the last several years. Multiple approaches have been taken to better understand the structural, molecular, and cellular events that underlie the progression from myocardial injury to myocardial infarction (MI) and, ultimately, to congestive heart failure. These approaches form the basis of the discussion of multimodality imaging that follows. SPECT techniques have come to the fore in animal models to characterize molecular markers such as matrix metalloproteinases (MMPs), angiotensin-converting enzyme (ACE), and angiotensin receptors that play important roles in left ventricular (LV) remodeling after MI. Angiogenesis, specifically the $\alpha_{\mathrm{v}} \beta_{3}$ integrin, is another important SPECT target. Cardiac MRI has made significant advances in targeting molecular events after MI such as apoptosis and

Received Sep. 28, 2009; revision accepted Feb. 3, 2010.

For correspondence or reprints contact: Christopher M. Kramer, University of Virginia Health System, Departments of Medicine and

Radiology, 1215 Lee St., Box 800170, Charlottesville, VA 22908.

E-mail: ckramer@virginia.edu

COPYRIGHT () 2010 by the Society of Nuclear Medicine, Inc. myeloperoxidase activity and cellular events including macrophage infiltration and collagen deposition. Diffusion tractography is a novel method used to describe changes in myofibrillar array after MI. Fluorescence tomography has been applied in mouse models to demonstrate macrophage infiltration and target molecular events. For characterizing mouse models of MI, cardiac MRI has evolved into the gold standard for the assessment of LV volumes, function, and infarct size. However, because of its high throughput and availability, echocardiography remains the workhorse in the field. Finally, the molecular imaging that is closest to clinical reality is SPECT of sympathetic innervation with ${ }^{123}$ I-metaiodobenzylguanidine (123I-MIBG). With additional clinical validation, this technique may become an important prognostic marker after MI and in congestive heart failure. More detail about and future challenges to these molecular imaging techniques are carefully outlined below.

\section{RADIOTRACER IMAGING FOR PREDICTION OF POSTINFARCTION LV REMODELING}

The changes that occur in structure, geometry, and eventually function of the left ventricle after MI have been termed post-MI remodeling. Ventricular remodeling is a complex biologic process that involves inflammation, angiogenesis, repair, and healing with specific biochemical and structural alterations in the myocardial infarct and periinfarct regions and remote regions $(1,2)$. It is now well recognized that the process of post-MI myocardial LV remodeling often leads to heart failure and is associated with important changes within the myocardial extracellular matrix. Disruption of the fibrillar extracellular matrix network results in a loss of normal structural support, resulting in myocyte fascicles being subjected to abnormal stress and strain patterns during the cardiac cycle, which in turn cause changes in myocardial geometry and function. MI results in the activation of the renin-angiotensinaldosterone system (RAAS), which in turn results in the activation of MMPs within the heart. The MMPs constitute a large family of proteolytic enzymes responsible for extracellular matrix degradation and remodeling under 
normal and pathologic conditions (3). A clear cause-andeffect relationship between MMPs and the LV remodeling process has been demonstrated through the use of animal models of developing congestive heart failure, transgenic models, and pharmacologic MMP inhibition studies (4-6). Recent clinical studies have also demonstrated the potential role of MMP activation in post-MI remodeling (7). However, noninvasive methods for detecting and quantifying the activation of the RAAS or MMPs in vivo during the evolution of post-MI remodeling have yet to be fully developed. These targeted imaging approaches will become critical for translating these basic observations to clinical applicability. Moreover, it is critical to define the regional relationship between LV geometry and mechanics to that of MMP or RAAS activity to move this field of post-MI biology from observational to mechanistic.

\section{Targeted Imaging of MMPs}

Investigators have been developing high-sensitivity MMP-targeted molecular imaging approaches for the evaluation of post-MI remodeling $(8,9)$. By radiolabeling molecules that target MMPs, such as pharmacologic inhibitors that specifically bind to the catalytic domain, one can visualize in vivo MMP activation after MI (Fig. 1) (9). Initial studies by $\mathrm{Su}$ et al. involved nonimaging techniques with an ${ }^{111}$ In-labeled broad-spectrum MMP inhibitor, ${ }^{111}$ InRP782, a molecule that selectively targets activated MMPs (9). This MMP-targeted agent demonstrated a favorable biodistribution in a murine model of MI, localized primarily within the infarct region, although a lesser increase in retention was also seen in the remote noninfarcted regions of the heart, consistent with global MMP activation and remodeling. Further imaging studies were performed using a ${ }^{99 m}$ Tc-labeled analog ( ${ }^{99 m}$ Tc-RP805) and hybrid SPECT/ CT with a dual-isotope protocol involving ${ }^{99 m}$ Tc-RP805 imaging and adjunctive ${ }^{201} \mathrm{Tl}$ perfusion imaging (10). The dual-isotope imaging studies revealed MMP activation within the perfusion defect region. These investigators have developed image-analysis tools to quantify regional ${ }^{99 \mathrm{~m}} \mathrm{Tc}$ RP805 from in vivo dual-isotope hybrid SPECT/CT images to track serial changes in MMP activation (Fig. 2) $(11,12)$. These dual-isotope imaging studies suggest that MMP activation is taking place within the sites of injury and remotely and support the concept that molecules that target MMP activation might be used to evaluate ventricular remodeling.

\section{Targeted Imaging with ACE Inhibitors and Angiotensin} Receptor I Antagonists

The RAAS plays an important role in regulating blood volume and systemic vascular resistance, which can influence cardiac output, systemic pressure, and local myocardial activity and therefore represent potential targets for molecular imaging of ventricular remodeling. Cardiac tissue levels of ACE and angiotensin type 1 and 2 receptors $\left(\mathrm{AT}_{1} \mathrm{R}\right.$ and $\mathrm{AT}_{2} \mathrm{R}$ ) have been measured in several studies of the RAAS in the heart and mediate the adverse effects of neurohormonal activation (13).
Several ACE inhibitors and $\mathrm{AT}_{1} \mathrm{R}$ antagonists have been radiolabeled for molecular imaging techniques (14). In a study of explanted hearts from patients with ischemic cardiomyopathy, ${ }^{18} \mathrm{~F}$-fluorobenzoyl-lisinopril was used to assess ACE levels in the myocardium (15). This study demonstrated that ${ }^{18} \mathrm{~F}$-fluorobenzoyl-lisinopril bound with some degree of specificity to areas adjacent to the infarct. Other studies using $\mathrm{AT}_{1} \mathrm{R}$ antagonists have demonstrated a differential between regional ACE activity and $\mathrm{AT}_{1} \mathrm{R}$ levels (16). In a murine model of $\mathrm{MI}$, investigators have used both a fluorescent angiotensin peptide analog and ${ }^{99 m}$ Tc-labeled losartan, an $\mathrm{AT}_{1} \mathrm{R}$ blocker for angiotensin receptor imaging. Uptake of the angiotensin-targeted fluorescent compound was seen in the infarct area at 1-12 wk after MI using an open chest imaging preparation, with maximum uptake within the heart being seen at approximately 3 wk after infarction. Subsequent histopathologic analyses suggested specificity for myofibroblasts within the infarct region in the weeks after the infarction (17). These investigators also performed in vivo micro-SPECT/CT with ${ }^{99 m}$ Tc-labeled losartan, demonstrating the feasibility for in vivo imaging of angiotensin receptor II in the heart (Fig. 3) (17).

These early studies suggest that the changes in the RAAS that take place within an infarction may be used to identify those at risk for developing significant heart failure after MI. To date, all of these studies have been performed in animal models, both small and large. Much more work is needed to assess the feasibility of these agents for the imaging of postinfarction remodeling in clinical trials.

\section{Correlation of Regional Activation of MMPs or RAAS with Regional Mechanics}

Targeted imaging of MMPs or the RAAS in combination with changes in LV deformation and geometry after MI using cardiac MRI or echocardiographic imaging methods may be useful to understand the mechanisms of post-MI remodeling.

This combined molecular-mechanical approach could help to establish whether the enhanced MMP activation that occurs after MI is associated with altered regional myocardial deformation and contributes to an acceleration of adverse remodeling. In addition, the combined approach could determine whether the risk for MMP-mediated postMI remodeling can be identified and tracked through novel multimodality noninvasive imaging of MMP or RAAS activation and LV deformation.

Studies are currently under way that use multimodality SPECT/CT and MRI or ultrasound imaging with established small- and large-animal models of post-MI remodeling to relate temporal changes in regional MMP activation to changes in regional myocardial deformation. Changes in MMP activation will also be related to associated changes in angiogenesis and activation of the RAAS. The targeted molecular imaging approach should provide unique insights 
A

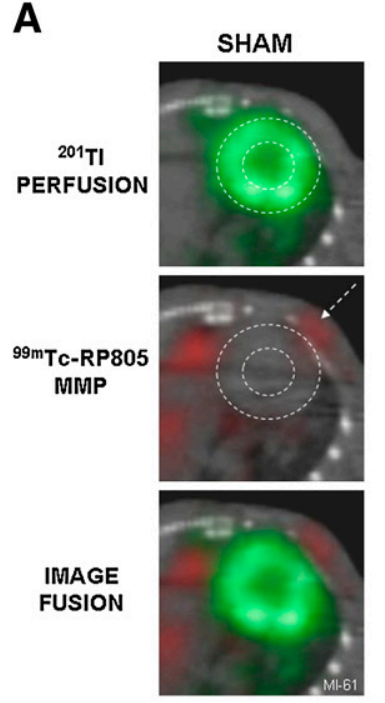

B
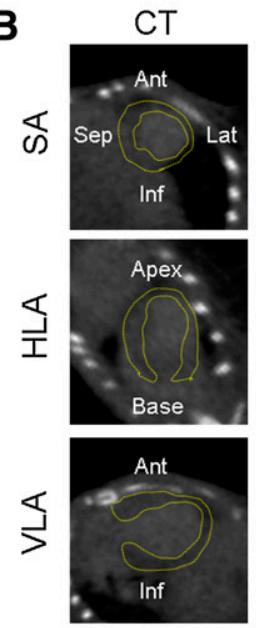

C
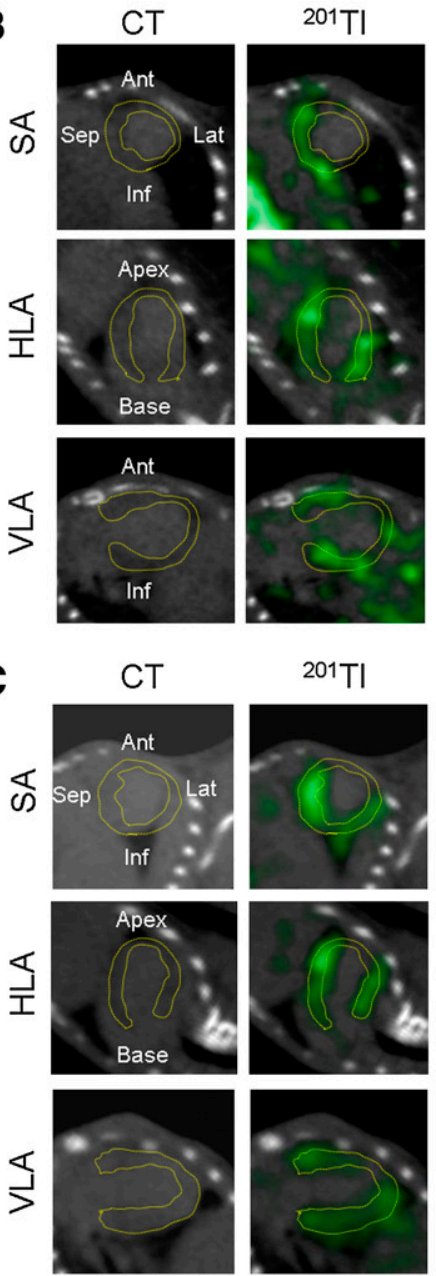

FIGURE 1. Imaging of MMP activity after infarction. Hybrid micro-SPECT/CT-reconstructed short-axis images were acquired without radiographic contrast $(A)$ in control shamoperated mouse (left) and selected mice at $1 \mathrm{wk}$ (middle) and 3 wk (right) after Ml, after injection of ${ }^{201} \mathrm{TI}$ (top row, green) and 99mTc-RP805 (middle row, red). Black-and-white and multicolor fusion image is shown on bottom. Control heart into the role of mechanical forces and MMP activation along with other critical molecular processes involved in ventricular remodeling and translate to direct clinical applications that hold both prognostic and diagnostic potential.

\section{Future Challenges and Directions for Radiotracer Imaging of Post-MI Remodeling}

An area of future investigation would be to address whether pharmacologic interventions known to favorably affect remodeling (i.e., ACE inhibitors or angiotensin receptor blockers) might influence regional tissue levels of MMPs and ACE or angiotensin receptors. This approach might predict an individual's response to pharmacologic therapy after MI. The specific application of target imaging of regional MMP activation as discussed holds the potential to directly quantify the extent and localization of MMP activation in vivo and relate these biologic events to the post-MI remodeling process. Recent studies have demonstrated a distinct spatial pattern of MMP activation with reference to the MI border and remote regions that change in a characteristic time-dependent manner after MI, highlighting the importance of tracking these regional and timedependent molecular changes (5). More importantly, these targeted noninvasive imaging approaches, if coupled with functional imaging, offer the opportunity to track novel therapeutic interventions directed at the reduction of postMI remodeling. However, several steps must take place before these molecular approaches are applied in the clinical setting. Food and Drug Administration approval of any of these molecular agents may take a great deal of time, effort, and expense.

\section{MRI AND FLUORESCENCE IMAGING OF MYOCARDIAL INJURY AND REMODELING}

MRI and fluorescence imaging techniques are frequently used to perform molecular imaging of biologic processes in the myocardium in small-animal models. Both techniques

demonstrates normal myocardial perfusion and no focal 99mTc-RP805 uptake within heart, although some uptake is seen in chest wall at thoracotomy site (dashed arrows). All post-MI mice have large anterolateral ${ }^{201} \mathrm{TI}$ perfusion defect

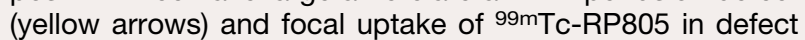
area. Dashed circle is drawn around heart to demonstrate localization of $99 \mathrm{mTc}$-RP805, MMP radiotracer, within infarcted area of heart. Some activity is also seen in periinfarct border zone. Additional micro-SPECT/CT images were acquired using higher-resolution SPECT detector after administration of radiographic contrast, at 1 (B) and 3 wk (C) after MI. Contrast agent permitted better definition of LV myocardium, which is highlighted by white dotted line. Representative short-axis (SA), horizontal long-axis (HLA), and vertical long-axis (VLA) images are shown. Focal uptake of $99 \mathrm{mTc}-\mathrm{RP} 805$ is seen within central infarct and periinfarct regions, which again correspond to ${ }^{201} \mathrm{TI}$ perfusion defect. Ant = anterior; Inf = inferior; Lat = lateral; Sep = septal. (Reprinted with permission of (9).) 


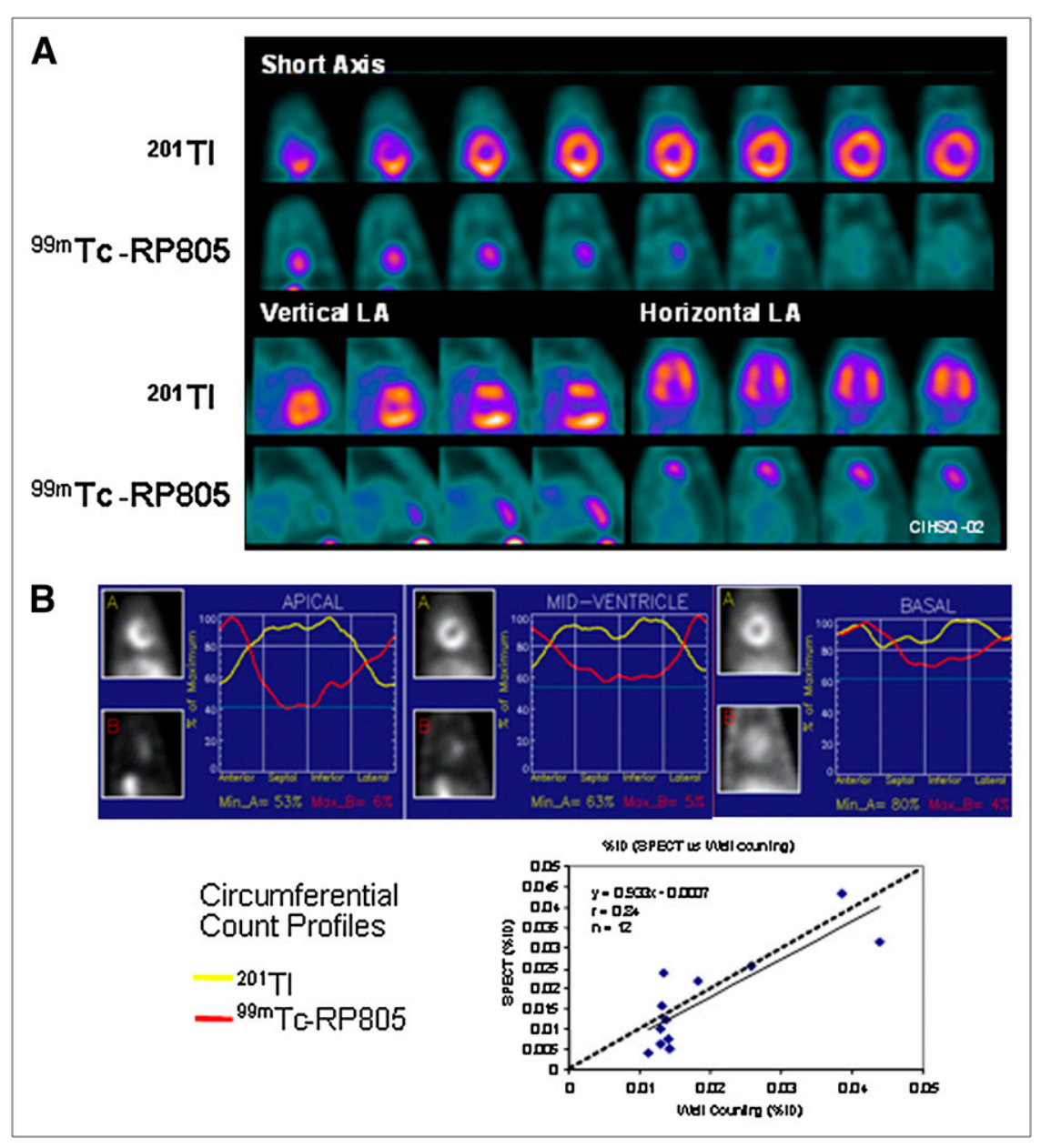

FIGURE 2. Quantification of hybrid dual-isotope 99mTc-RP805 and ${ }^{201} \mathrm{~T}$ SPECT/CT images. (A) Registered dual ${ }^{201} \mathrm{Tl} /{ }^{99 m} \mathrm{Tc}-\mathrm{RP} 805$ SPECT/CT-based attenuation-corrected images in a dog 2 wk after $\mathrm{Ml}$ are shown in standard format. Images were quantified using circumferential quantitative approach previously described. Activity (percentage injected dose [\%ID]) quantified from dual $201 \mathrm{Tl} / 99 \mathrm{mTC}$-RP805 SPECT with attenuation correction using global minimum normal limit was compared with activity (\%ID) derived from postmortem $\gamma$-well-counting. (B) Quantitative profiles and summary of quantitative data for same dog as in A. LA = long axis. (Adapted with permission of (12).)

are nonionizing, provide multiple contrast mechanisms, and are able to image the myocardium across several spatial scales. Traditionally, MRI has been thought of as a wholeorgan imaging modality, providing highly accurate measurements of indices such as LV mass, ejection fraction, and infarct size. Fluorescence imaging, on the other hand, has traditionally been regarded as an in vitro tool for flow cytometry and microscopy. However, recent advances in imaging hardware, contrast agents, acquisition schemes, and reconstruction algorithms have allowed these respective modalities to move toward each other and into new imaging domains. Diffusion-encoded MRI techniques have been developed to image 3-dimensional myocardial microstructure, and a range of molecular MRI agents now allow cellular and subcellular processes in the myocardium to be imaged noninvasively in vivo, again in small-animal models.

FIGURE 3. Noninvasive imaging of angiotensin receptors with radiolabeled losartan. Micro-SPECT and micro-CT images are shown in control mouse after $99 \mathrm{mTc}$-losartan administration; no uptake in heart can be seen in in vivo and ex vivo images (A). There is only some liver uptake, on bottom left of SPECT image. (B) In 3-wk post-MI animal, significant radiolabeled losartan uptake is observed in anterolateral wall (arrows). Infarct uptake on in vivo image is confirmed in ex vivo image. Histo-

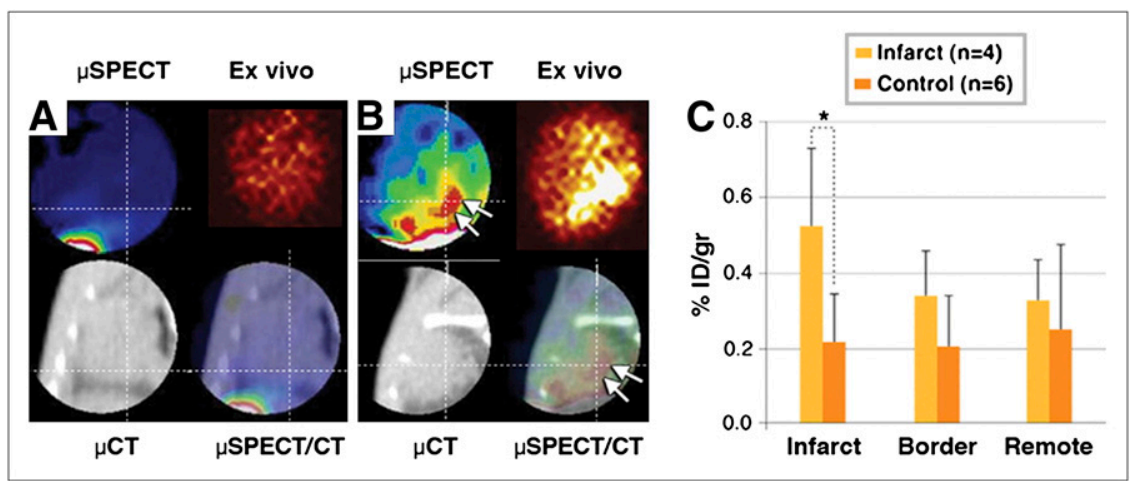
gram (C) demonstrates significantly (*) higher uptake in infarcted region $(0.524 \pm 0.212$ percentage injected dose per gram [\%ID/g]), as compared with control noninfarcted animals $(0.215 \pm 0.129 \% \mathrm{ID} / \mathrm{g} ; P<0.05)$. (Reprinted with permission of (17).) 
Optical projection tomography (OPT) allows 3-dimensional fluorescence datasets of myocardial microstructure to be obtained, and fluorescence molecular tomography (FMT) can be used to image fluorescent signatures in the myocardium in small animals noninvasively in vivo. Although a detailed description of each of these techniques is beyond the scope of this article, the central technical advances and applications of these techniques are in the following sections.

\section{Diffusion MRI of Myocardial Microstructure}

The myocardium has a complex 3-dimensional microstructure and consists of myofiber bundles arranged in an array of crossing helices $(18,19)$. In the mid myocardium, muscle fibers curve around the long axis of the left ventricle circumferentially and have a neutral helix angle. In the subendocardium, however, the myofibers have a positive or right-handed helix angle and in the subepicardium a negative or left-handed helix angle. This microstructural pattern is critical for optimal cardiac function and becomes perturbed in diseased myocardium (20). Although much information can be gained from histologic examination of the myocardium, the development and application of noninvasive techniques capable of imaging myofiber architecture noninvasively would be a significant advance (21). Such imaging tools would allow changes in myofiber architecture to be followed serially and be correlated with cellular and molecular changes in the myocardium. The imaging of myocardial microstructure thus spans the spatial scale, linking molecular events to whole-organ pathophysiology.

Water molecules diffuse along the long axis of myofibers and produce a phase change in the MRI signal in the presence of a diffusion-encoding gradient that is applied parallel to the direction of the myofibers (22). Several formalisms have been developed to resolve the diffusion of water, and hence myofiber orientation, in a diffusionencoded MRI acquisition (22,23). The most robust of these techniques is an approach known as diffusion spectrum MRI (or DSI) (22-24), which has been used recently to image myocardial microstructure (25). The properties of DSI support the generation of continuous 3-dimensional tractograms of myofiber architecture, as shown in the normal rat heart in Figure 4 (25). DSI tractography has also been used to resolve changes in myocardial microstructure after MI in rats (25). Three weeks after infarction, a significant number of residual myofibers persisted within the infarcts. The residual myofibers consisted of subendocardial, mid-myocardial, and subepicardial fibers and frequently intersected or made contact with each other much like fibers in a mesh. Ongoing work with this technique is focusing on its application in large-animal models of cardiac disease and its correlation with mechanical indices of cardiac function. DSI tractography and other diffusionbased imaging techniques thus provide a valuable mechanistic bridge between events at the molecular level and changes in whole-organ function. The integration of molecular and microstructural MRI into a single integrated and comprehensive MRI dataset would be extremely powerful and is being pursued actively in small-animal models.

\section{Molecular MRI of Myocardium}

Molecular MRI is in many ways extremely suited to the imaging of the myocardium in mice (26). The technique provides high-resolution molecular readouts that are integrated with the functional and physiologic information provided by MRI. The principal challenge of performing molecular MRI in the myocardium, however, is the need to achieve adequate sensitivity. This is significantly more challenging with MRI, which has a relatively low sensitivity (26).

Two strategies have been used to address the issue of sensitivity. The first involves the use of conventional gadolinium chelates, with micromolar sensitivity, to image highly expressed targets in the myocardium. The prototype agent in this class is a collagen-binding gadolinium chelate, which binds to the abundantly expressed type 1 collagen in the myocardium (27). In normal myocardium, the binding of the probe to interstitial collagen can be used to image myocardial perfusion (28), and in the presence of prior infarction the agent binds specifically to myocardial scarring (29). Translation of this strategy into the clinical arena is simplified by the well-known pharmacokinetics and toxicities of conventional gadolinium chelates. However, numerous molecular targets of interest in the myocardium are in the nanomolar range and are thus not detectable with this strategy.

A second molecular MRI approach has been developed to image sparsely expressed targets. This approach involves the use of high-relaxivity compounds with nanomolar sensitivity (26). Targeted liposomes loaded with large amounts of gadolinium are frequently used as high-relaxivity compounds in the vasculature but are not suited to the imaging of myocardial targets because of their size and inability to cross the capillary membrane. Superparamagnetic iron-oxide nanoparticles (MNPs), however, are ideally suited to the imaging of sparse molecular targets in the myocardium $(26,30)$. These agents are small $(30-50 \mathrm{~nm})$, have high magnetic relaxivities, and have, in the case of long-circulating agents, a thick dextran coat designed to make the particle biologically inert (30). In ischemic injury, the capillary membrane becomes hyperpermeable, and MNPs cross the capillary membrane rapidly and in large amounts (31), thus reaching the target of interest in the interstitial space of the myocardium (32). MNPs, however, are also able to cross completely normal capillary membranes via diffusion and other slow transport mechanisms. This ability to cross membranes has allowed MNPs to be used to image lymph node metastases (33) and targets in chronic heart failure where the capillary membrane is completely normal (34). The inert nature of these long-circulating MNPs prevents them from reacting 
FIGURE 4. DSI tractography of excised rat heart showing transmural variation in myocardial microstructure (25). Fiber tracts are color-coded by helix or spiral angle they make with long axis of left ventricle. Left ventricle is being viewed from its lateral wall ( $A$ and $\mathrm{C}-\mathrm{F}$ ) and in its short axis (B). Only those fibers intersecting spheric region of interest are displayed in D-F. Subendocardial fibers have positive or righthanded helix angle, and in lateral wall, they course toward anteroapex. However, those in subepicardium have negative or left-handed helix angle, and in lateral wall, they course from
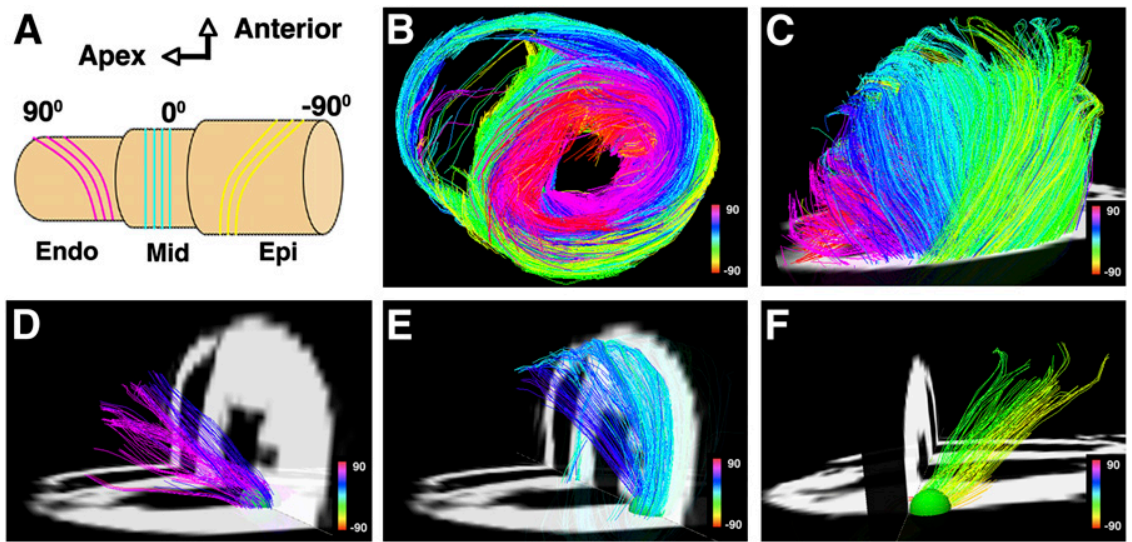

anterobase toward posteroapex. Fibers in mid myocardium have a zero helix angle and are thus circumferential. Endo $=$ endocardium; Mid = mid myocardium; Epi = epicardium. (Reproduced with permission of (23).)

nonspecifically in the complex milieu of the interstitial space of the myocardium. In their unmodified form, the blood half-life of MNPs in mice is approximately $12 \mathrm{~h}$. The conjugation of a ligand, whether a peptide or small protein, to the MNP decreases its circulatory half-life in mice to less than $3 \mathrm{~h}(32,35)$. This decrease is in many ways advantageous because the circulatory time is still adequate for delivery of the MNP to the interstitial space of the myocardium but allows acute processes to be imaged with less background signal (32). MNPs possess another important characteristic that aids in their detection. On binding to the cell surface, targeted MNPs are internalized and aggregate in lysosomes, increasing their detectability even further because a nanoassembly of MNPs has a far higher transverse relaxivity than the same amount of the agent in a monodisperse form (36).

MNPs are avidly taken up by macrophages and can be used to image myocardial inflammation in ischemic heart disease, transplant rejection, and other inflammatory conditions in mouse models. Within 24-72 $\mathrm{h}$ of infarction, the myocardium becomes heavily infiltrated with highly proteolytic macrophages (37). The macrophage infiltrate persists for approximately 2 wk but becomes less proteolytic by the end of the first week (37). MNPs have been used to image this macrophage infiltration in mice after MI with both conventional gradient-echo MRI techniques and offresonance techniques $(38,39)$. Gradient-echo MRI is T2*weighted and exploits the ability of the high transverse relaxivity of the MNP to significantly reduce the intensity of the MRI signal. Signal hypointensity is thus produced in the vicinity of the MNP (Fig. 5). Conventional T2* imaging is sensitive, shows a linear response to the dose of MNP, and correlates well with the fluorescent readout from the probe (39). Concerns have been raised, however, about the specificity of negative MRI contrast. Several novel techniques have thus been developed to generate positive contrast from MNP. Most of these techniques have been tested in the imaging of MNP-labeled stem cells. A positive-contrast off-resonance technique, however, has been tested in a mouse model of MI (38). Positive contrast was seen in areas of the myocardium infiltrated with macrophages (Fig. 5), but the technique was less sensitive and less linear than the conventional $\mathrm{T} 2 *$-weighted gradient-echo approach (38). Nevertheless, off-resonance and other positive-contrast approaches provide a valuable additional contrast mechanism and demonstrate the breadth and flexibility of contrast mechanisms available in molecular MRI.

Targeted imaging with MNP in the myocardium cannot be performed in the presence of a dense macrophage infiltrate. In ischemic heart disease, imaging with targeted MNPs must thus be performed less than $24 \mathrm{~h}$ or more than $14 \mathrm{~d}$ after injury. Likewise, models of heart failure that are not characterized by a dense macrophage infiltrate need to be used for targeted imaging with MNP (34). These 2 strategies have been used to image cardiomyocyte apoptosis in the heart, with the apoptosis sensing MNP AnxCLIO-Cy5.5 $(32,34,40)$. The annexin moiety on the probe binds to phosphatidylserine on the surface of apoptotic cells, cross-linked iron-oxide (CLIO) provides an MRI readout, and Cy5.5 allows fluorescence imaging of the agent to be performed. In an initial study in a mouse model of ischemic reperfusion, AnxCLIO-Cy5.5 was able to image cardiomyocyte apoptosis in vivo (Fig. 5) (40). Uptake of the agent was seen only in areas of injured and hypokinetic myocardium, and no significant uptake of the control agent was seen. Probe uptake was quantified by the construction of MRI relaxation maps in vivo (Fig. 5), and probe uptake could be confirmed by fluorescence imaging of the Cy5.5 moiety in the injured myocardium (40). In a recent follow-up study, AnxCLIO-Cy5.5 was used in conjunction with the delayed enhancement of a novel gadolinium chelate to simultaneously image cardiomyocyte apoptosis and necrosis in ischemic myocardium (32). Large areas of apoptotic but viable (delayed enhancement-negative) myocardium were present in the mid myocardium within the first few hours of ischemic injury (32). 

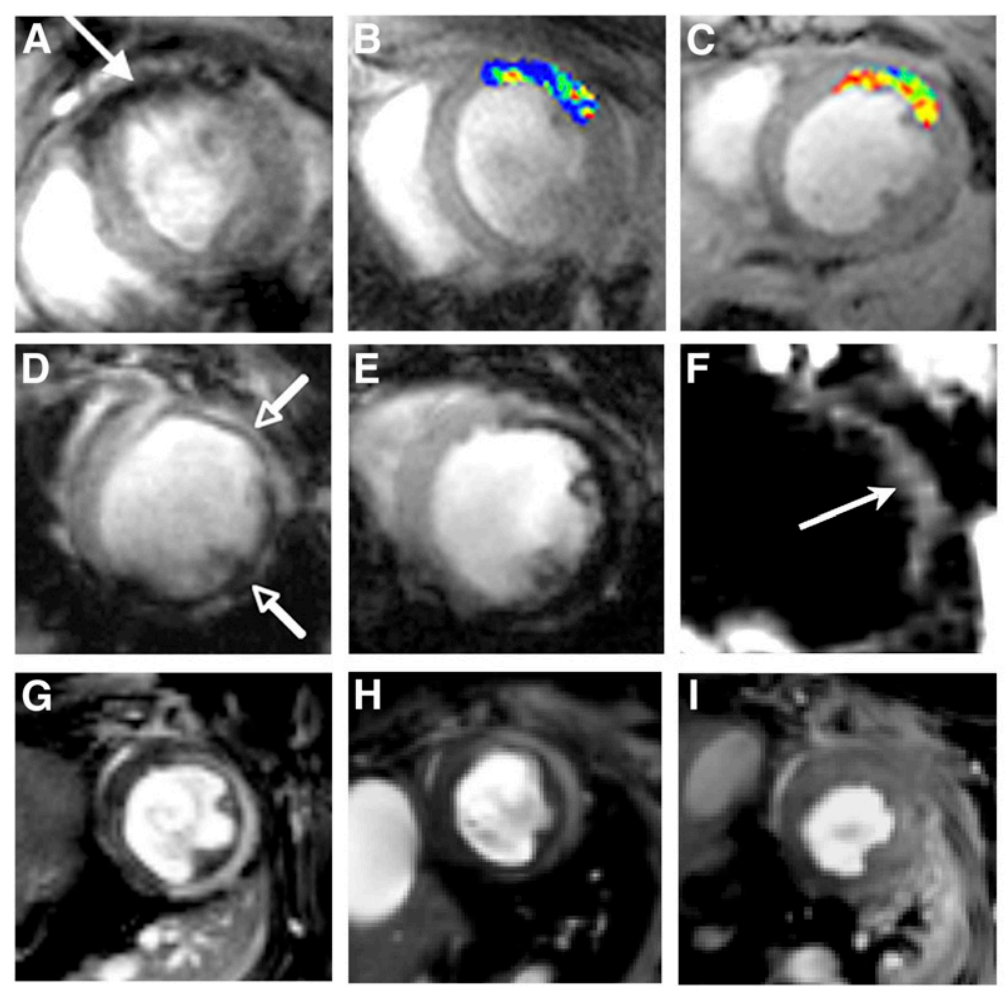

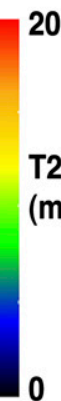

T2*

(ms)

FIGURE 5. Molecular and cellular MRI of myocardium in vivo in mice with ischemic myocardial injury. $(A-C)$ In vivo MRI of cardiomyocyte apoptosis in mouse model of ischemic reperfusion (40). (A) Uptake of AnxCLIO-Cy5.5 in injured myocardium produces negative contrast in anterior wall. (B and $\mathrm{C}$ ) $\mathrm{T}^{*}$ * maps created in hypokinetic areas of myocardium. T2* was significantly shorter in mice injected with active probe (B) than in mice injected with unlabeled control probe (C). (Reproduced with permission of (40).) ( $D$ and E) In vivo MRI of myocardial macrophage accumulation in mice $96 \mathrm{~h}$ after MI. (Reproduced with permission of (39).) Mouse in D was injected intravenously with $3 \mathrm{mg}$ of CLIO-Cy5.5 per kilogram and mouse in $\mathrm{E}$ with $20 \mathrm{mg}$ of CLIO-Cy5.5 per kilogram. Linear response in tissue contrast between uninjured septum and injured anterolateral wall (arrows) is seen (39). (F) Offresonance imaging of postinfarction myocardial macrophage infiltration (38). Positive contrast is produced in vicinity of iron-oxide uptake (arrow) but also in other areas of susceptibility shifts such as air-tissue interfaces. (Reproduced with permission of (38).) (G-I) In vivo MRI of myocardial myeloperoxidase activity in healing myocardial infarcts. (Reproduced with permission of (42).) (G) Wild-type mouse injected with myeloperoxidase-activatable agent shows increased signal intensity in injured anterolateral wall on T1-weighted image. Significantly less signal enhancement is seen in heterozygous myeloperoxidase knockout mouse $(H)$ and virtually none in homozygous knockout mouse (I) (42).

Examples of cellular (macrophage infiltration) and molecular (cardiomyocyte apoptosis) imaging were described earlier in this article. The armamentarium of molecular MRI, however, is currently being extended further with activatable and multispectral imaging approaches. Activatable MRI agents undergo a conformational change in response to their environment or because of activation by an enzyme, causing the magnetic relaxivity of the imaging agent to increase, enhancing its detection $(26,36)$. Activatable MNP agents, also known as magnetic relaxation switches, are well suited to in vitro diagnostics (multiplexed miniaturized point-of-care, or "laboratory on a chip" diagnostics), but their use in vivo is at present not feasible (26,36). Activatable gadolinium-based agents are less suited to in vitro diagnostics but can be used to detect enzyme activity in vivo. A gadolinium-serotonin chelate, for instance, has been used to image myeloperoxidase activity in vivo in mice (41). In the presence of myeloperoxidase, serotonin is oxidized, causing the chelate to form dimers and oligomers with higher relaxivity. Proteolytic macrophages infiltrating a myocardial infarct produce large amounts of myeloperoxidase that can be readily imaged in vivo with this construct (Fig. 5) (42). Because gadolinium chelates are small, most organic fluorochromes cannot be attached to them without drastically changing their properties (32). The cellular specificity of targeted gadolinium- based agents thus frequently needs to be determined by scrambling the ligand on the probe (29) with competitive inhibition strategies or through the use of transgenic mouse models. For instance, as shown in Figure 5, no signal enhancement was seen in the infarcts of homozygous knockout myeloperoxidase mice injected with the myeloperoxidase activatable agent, confirming the specificity of the agent for myeloperoxidase (42).

MNP, in contrast to gadolinium chelates, can be easily labeled with fluorochromes to yield magnetofluorescent nanoparticles such as AnxCLIO-Cy5.5. The fluorescent moiety on the magnetofluorescent nanoparticles allows flow cytometry and fluorescence microscopy of the agent to be performed in vitro and also allows the nanoparticle to be imaged in vivo with novel noninvasive imaging techniques such as fluorescence tomography and OPT.

\section{Fluorescence Tomography of Myocardium}

Fluorescence imaging of deep structures, such as the heart, requires light at both the illumination and emission wavelengths to be able to penetrate tissue. Light in the visible spectrum, however, is highly absorbed by tissue and penetrates less than $1 \mathrm{~mm}$. Light in the near-infrared (NIR) portion of the spectrum is minimally absorbed and can penetrate several centimeters into tissue (43). This allows fluorescence imaging of the heart to be performed 
noninvasively in small-animal models. Fluorescence imaging of the human heart, though, requires invasive or catheter-based approaches. Tissue autofluorescence is lowest in the NIR portion of the spectrum, adding further value to NIR imaging. The greatest experience with FMT to date has thus been with organic NIR fluorochromes (44), although the development of quantum dots that are both excited and emit in the NIR spectrum is being pursued actively. Organic NIR fluorochromes such as Cy5.5 can be imaged in vivo as part of a larger MNFP, such as AnxCLIO-Cy5.5, or be a component of smaller enzyme-activatable NIR fluorochromes (44). Both strategies have been used with success in the myocardium.

FMT of the myocardium in small animals has been made possible not only by the development of NIR fluorochromes but also by novel detector and reconstruction algorithms (45). While the propagation of $\mathrm{x}$-ray radiation through tissue is influenced primarily by the $\mathrm{x}$-ray absorption coefficient of tissue, the propagation of light in tissues is diffuse and is a function of both absorption and scattering $(43,45)$. During current FMT acquisitions, the mouse is rotated and thus illuminated in numerous source-detector configurations over $360^{\circ}(46)$. A surface image of the mouse is acquired to compensate for reflection and refraction at surface interfaces. FMT reconstruction algorithms yield the distribution of the fluorochrome in the tissue from the detected emitted fluorescence.

The initial application of FMT in the myocardium involved the detection of myocardial macrophage infiltration in mice with MI (Fig. 6) (39). The infarcted mice were injected with CLIO-Cy5.5 and imaged $96 \mathrm{~h}$ after infarction. The detection of the macrophage infiltrate from the magnetic signal produced by the iron-oxide component of the probe has been discussed. FMT of the Cy5.5 moiety on the probe proved equally accurate and, like T2*-weighted MRI, had a highly linear response to increasing doses of the probe (39). In a subsequent study, FMT was used to image a cathepsin-activatable NIR fluorochrome in healing myo- cardial infarcts (47). The macrophages infiltrating the infarct secrete large amounts of cathepsin, which cleaves a recognition site on the linker portion of the probe. After cleavage, the Cy5.5 moieties on the probe-which are held near each other and thus quench-separate and fluoresce (44). This basic principle can be applied to the generation of numerous enzyme-activatable NIR fluorochromes. A linker cleavable by MMPs, for instance, has been used to image the time course of MMP activity in the heart after MI (8).

FMT is a completely noninvasive and quantitative technique that can easily be performed serially in mice. However, the high-spatial-resolution characteristic of fluorescence microscopy is lost with FMT, which has a spatial resolution of approximately $1 \mathrm{~mm}$ (45). An intermediate regime maintaining attributes of both FMT and fluorescence microscopy has thus been developed. OPT is a technique that images the entire mouse heart at the meso- and microstructural levels $(48,49)$, much like diffusion MRI. Although the technique can be performed on whole organs such as the heart, it cannot be performed in vivo. OPT involves chemical treatment of the excised tissues to eliminate those structures causing scattering. The propagation of light through tissue can then be approximated purely as a function of attenuation, and the image data can be reconstructed much like CT images (inverse radon transform) $(48,49)$. OPT allows microstructural details such as trabeculations and valve leaflets in the mouse heart to be visualized in the intact heart (Fig. 7) (48). High-resolution fluorescence tomographic datasets can also be obtained with this approach.

Future Challenges and Directions for MRI and Fluorescence Imaging of Myocardial Injury and Remodeling

Fluorescence and MRI techniques are highly complementary. Fluorescence imaging is extremely sensitive and highly multispectral, and molecular MRI provides highresolution molecular images that can be correlated with MR images of myocardial structure and function. Although the clinical translation of both NIR fluorochromes and molecular
FIGURE 6. FMT of myocardial macrophage infiltration in vivo (39). Reconstructed coronal slices from 3-dimensional FMT dataset have been superimposed on white-light images of mice. Slices $2-4$ in FMT dataset intersected heart, whereas slices 5-8 passed posterior to it. (A) Long-axis $\mathrm{MRI}$ in infarcted mouse corresponding to slice 2 from fluorescence dataset of that mouse, which passed through heart (B). (C) Slice 5 from fluorescence dataset of infarcted mouse, which passed posterior to heart. Corresponding slices (D, slice 2; E, slice 5) of sham-operated mouse are shown. (F) Depth-resolved fluorescence intensity in heart was significantly greater $\left({ }^{\star} P<0.05\right)$ in infarcted mice than in sham-operated mice. AU $=$ arbitrary units. (Reproduced with permission of (39).)

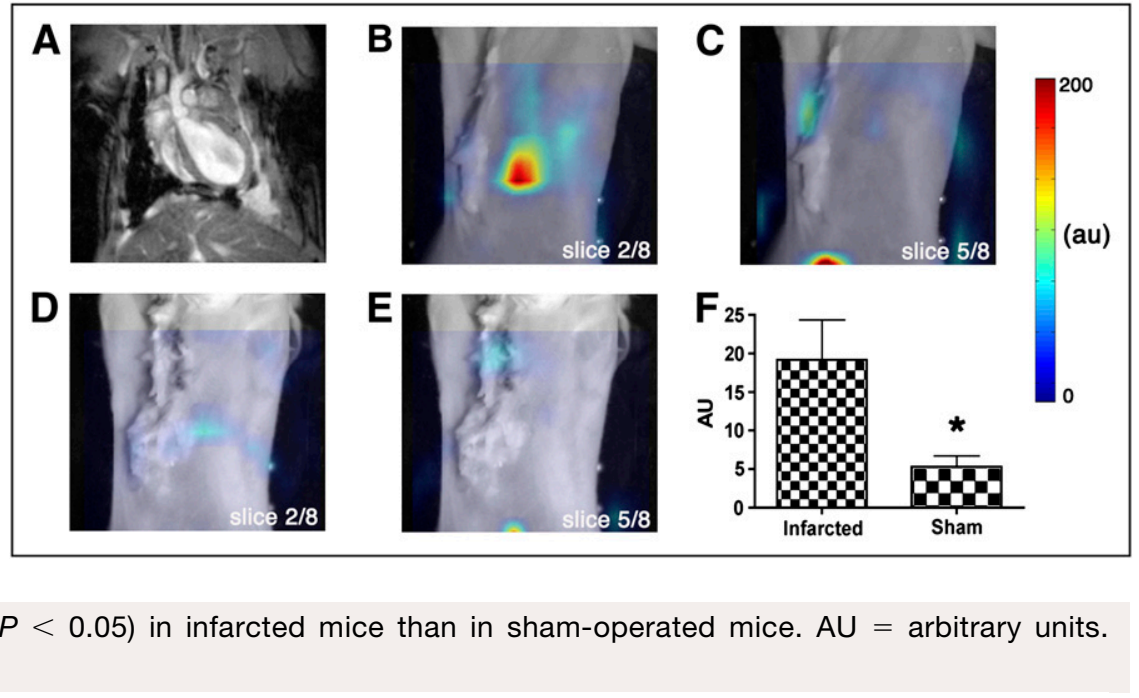



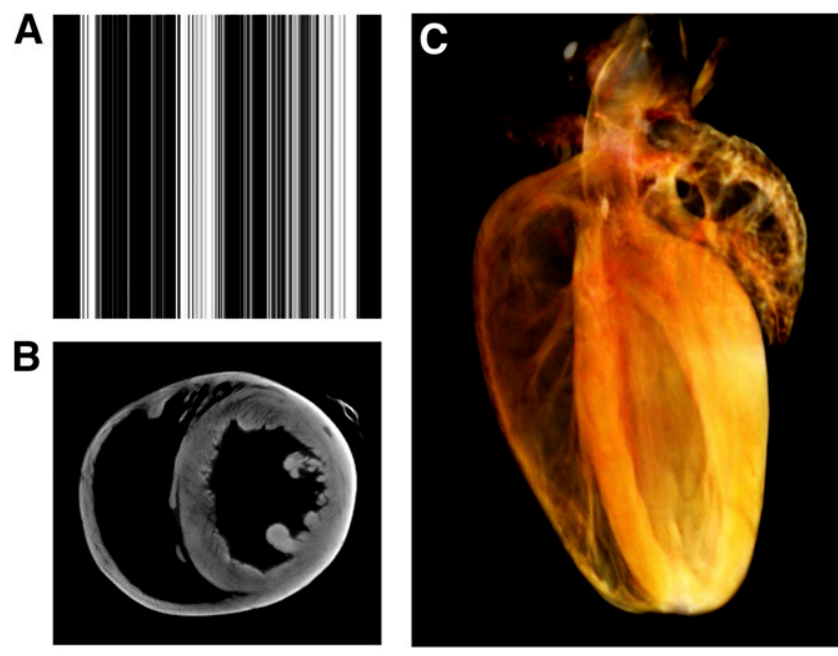

FIGURE 7. OPT of mouse heart ex vivo (49). (A) Chemical treatment of heart reduces scattering, allowing tomographic reconstruction to be produced purely from absorption maps of tissue. (B) Short-axis reconstruction of mouse heart produced by inverse radon transform of absorption data. (C) Volume-rendered OPT reconstruction of mouse heart showing structures such as trabeculations in fine detail. (Reproduced with permission of (49).)

MRI agents is more complex than that of radiolabeled imaging agents, NIR fluorochromes such as indocyanine green are already used clinically and 2 MNPs (ferumoxides [Advanced Magnetics] and ferumoxytol [Advanced Magnetics]) have been approved for clinical use in the United States. Molecular and microstructural MR and fluorescence imaging techniques will continue to evolve and will likely become valuable tools in both preclinical and clinical medicine, although the former is close to realization and the latter will require significant advances before coming to fruition.

\section{MULTIMODALITY IMAGING OF MYOCARDIUM AFTER MI}

The widespread availability of transgenic and knockout mice, along with the recent sequencing of the mouse and human genomes, provides biomedical researchers with unique opportunities to more fully elucidate the molecular mechanisms underlying cardiovascular disease. Indeed, the widespread use of mice to study human cardiovascular disease has created a demand for noninvasive imaging modalities capable of accurately assessing cardiac anatomy, function, and biochemistry. Thus, a common goal shared by many cardiovascular imaging programs is to integrate quantitative functional imaging, molecular imaging, and image analysis to better understand the complex molecular, structural, and functional events initiated by MI in mice. Several imaging modalities have been explored for this purpose, including MRI, ultrasound, PET, SPECT, CT, and optical imaging modalities.

\section{Structural and Functional Imaging}

Cardiac MRI. Cardiac MRI is widely acknowledged as the gold standard for serially assessing the response of the murine heart to surgically induced MI. Three-dimensional cardiac anatomy can be assessed in detail using multislice cine MRI on high-field scanners. Furthermore, late gadolinium enhancement can be used to accurately measure the size of MI with cardiac MRI as early as $1 \mathrm{~h}$ after reperfusion in mice (50). The ability of cardiac MRI to serially assess LV volumes, ejection fraction, and wall thickening in mice provides investigators with insight into the biomechanical mechanisms underlying LV remodeling after MI (51). Furthermore, tissue-tracking techniques such as myocardial tagging or displacement-encoded imaging can be used to quantify regional intramyocardial function at high spatial resolutions on high-field scanners (Fig. 8) (52,53). Although some cardiac MRI techniques work as well in mice as they do in humans, the high heart rate in mice ( $\sim 600$ beats per minute) can pose challenges in adopting certain clinical pulse sequences, such as inversion-recovery imaging, in the mouse (54). Another challenging example is applying T2-weighted imaging to detect edematous regions in the heart after MI (55). This region of edema is hypothesized to demarcate the ischemic area at risk for infarction and thus may prove valuable in the
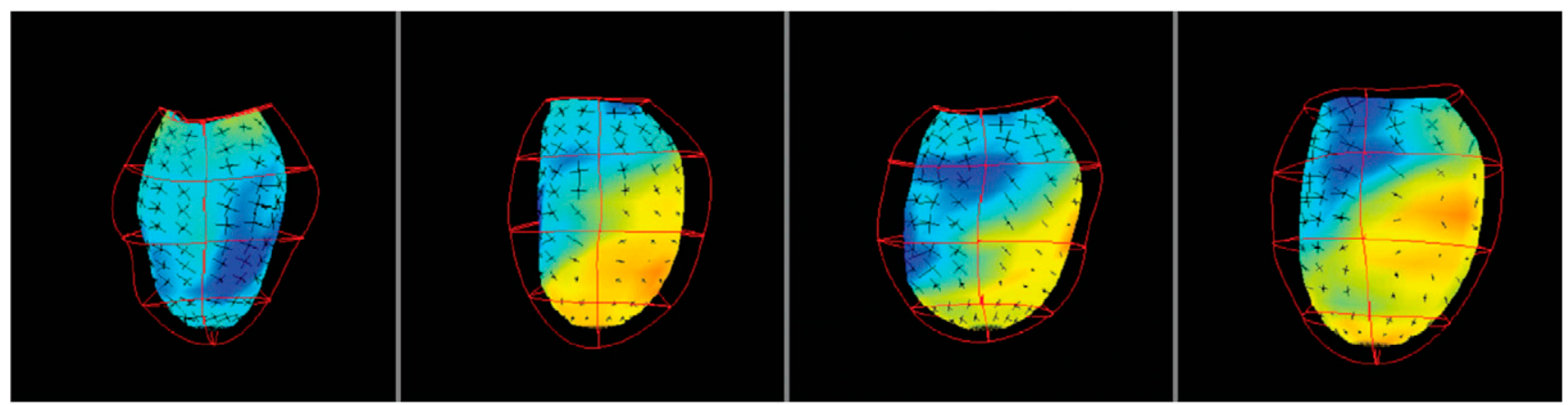

FIGURE 8. Three-dimensional finite element model derived from 3-dimensional myocardial tagging cardiac MRI study of LV remodeling in mice after reperfused MI. Each panel depicts end systole, at baseline and at days 1, 7, and 28 after MI (from left to right). Septum is on left. Lines denote model element boundaries. Crosses denote 3-dimensional principal strains and directions. (Adapted with permission of (53).) 
noninvasive measurement of infarct size as a function of area at risk. In other applications, clinical pulse sequences that are difficult to implement in the human heart, such as arterial spin labeling, are actually easier to implement in mice because of the short R-R interval (56). The addition of T2-weighted imaging and arterial spin labeling to the cardiac MRI toolkit makes it the "1-stop shop" (57) for measuring most every parameter necessary to noninvasively assess acute MI and post-MI LV remodeling in the murine heart (i.e., myocardial perfusion, wall thickening, intramyocardial strain, area at risk, infarct size, LV volumes, and myocardial mass). The serial assessment of these functional parameters at high resolution with cardiac MRI in transgenic and knockout mice has already provided new insights into the role of individual genes in the process of post-MI LV remodeling (58-61).

Ultrasound. Although cardiac MRI is widely considered to be the gold standard of cardiac imaging, the widespread availability of ultrasound equipment makes ultrasound the single most widely used modality for imaging cardiac function in mice. The cost of acquiring, siting, and maintaining an ultrasound scanner is only a small fraction of that required for an MRI scanner. Furthermore, the superior temporal resolution available on many ultrasound scanners at least partially offsets the advantage in spatial resolution enjoyed by high-field MRI scanners. Because ultrasound is the default cardiac imaging modality at most institutions, a fair amount of effort has been invested in developing ultrasound instrumentation suitable for murine cardiac imaging (62) and in validating ultrasound endpoints against cardiac MRI. For example, 3-dimensional cardiac anatomy can be assessed by ultrasound using a motion stage (63), and ultrasound speckle-tracking techniques can be used to measure intramyocardial strain at resolutions comparable to cardiac MRI using displacement encoding with stimulated echoes (Fig. 9) (64). Furthermore, myocardial contrast echocardiography can be used to assess the area at risk in the murine heart (65) and regional differences in perfusion (Fig. 10). Currently, ultrasound lacks a definitive method for the direct determination of infarct size. Nevertheless, the absence of wall thickening (or strain generation) can be used as a reliable surrogate endpoint in most studies, except those in which chronic partial coronary occlusions or multiple transient occlusions are being used to induce myocardial hibernation. In the final analysis, ultrasound has been used in many studies that use imaging to assess cardiac phenotype in transgenic or knockout mice, and ultrasound will continue to be the default imaging modality for determining such functional parameters for many years to come.

\section{Molecular and Cellular Imaging}

Cardiac MRI and Ultrasound. Cardiac MRI and ultrasound can also be used for molecular imaging of biologic processes critical to the pathophysiology or treatment of ischemic heart disease. Examples include using cardiac MRI to track the infiltration of iron-oxide-tagged macro-
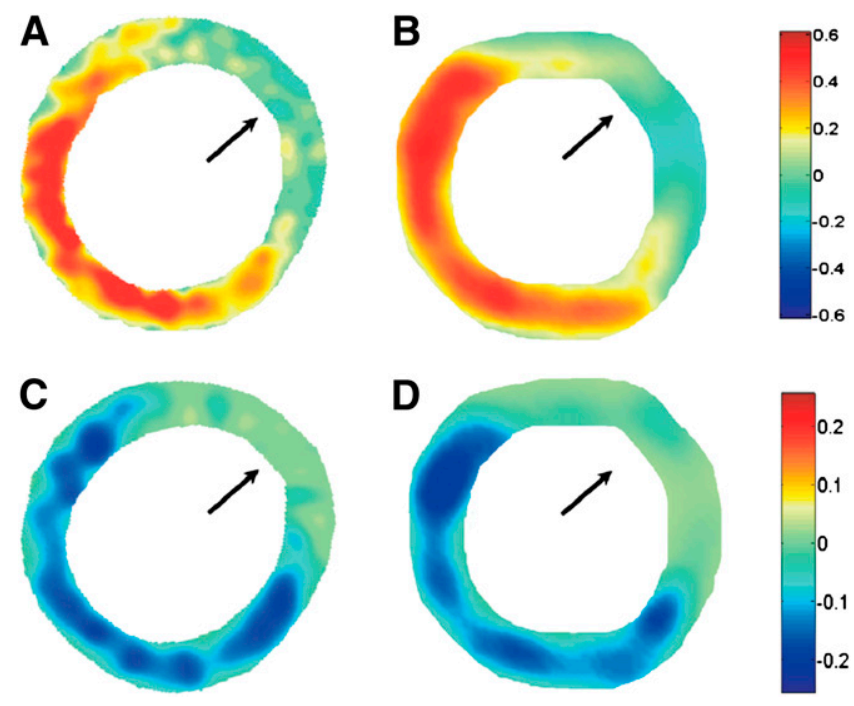

FIGURE 9. End-diastolic-to-end-systolic radial strain maps from mouse heart $1 \mathrm{~d}$ after reperfused MI using displacementencoding-with-stimulated-echoes cardiac MRI (A) and speckle-tracking analysis of ultrasound images (B). Enddiastolic-to-end-systolic circumferential strain maps from same mouse heart using cardiac MRI (C) and ultrasound (D). In both short-axis radial and circumferential maps, defects in contraction are observed in anterolateral LV (as indicated by arrows). (Adapted with permission of (64)).

phages into the heart after MI (39), iron-oxide to monitor stem cell therapy (66), and gadolinium-tagged imaging probes to quantify collagen deposition in the infarct zone after MI (29). Previously ischemic regions of tissue can be detected by ultrasound using microbubbles targeted to activated leukocytes (67) or to the activated endothelium (68). However, in these applications, the relatively low sensitivity of MRI often limits the utility of MRI contrast agents to the detection of fairly abundant targets, and the molecularly targeted microbubble contrast agents used by ultrasound are largely confined to the intravascular space.

PET and SPECT in Small Animals. In contrast, PET and SPECT provide for the highly sensitive detection of radioactive probes in murine models, as previously discussed. From the standpoint of general utility, gated PET and SPECT can also be used to assess cardiac function in mice $(69,70)$. Furthermore, PET has long been considered the gold standard for the assessment of myocardial viability, although SPECT is more commonly used in clinical applications. One recent study used PET (with ${ }^{18} \mathrm{~F}-\mathrm{FDG}$ ) and SPECT (with ${ }^{99 \mathrm{~m} T c-m e t h o x y i s o b u t y l i s o n i t r i l e) ~ t o ~ n o n-~}$ invasively assess viability in a rat model of MI, as compared with late gadolinium-enhanced cardiac MRI (71). Thus, although PET and SPECT may not be frontline systems for measuring cardiac function in mice because of their limited resolution, they are nevertheless quite capable of measuring chamber volumes, perfusion, and viability. These functional imaging capabilities, combined with their exquisite sensitivity for detecting molecularly targeted 


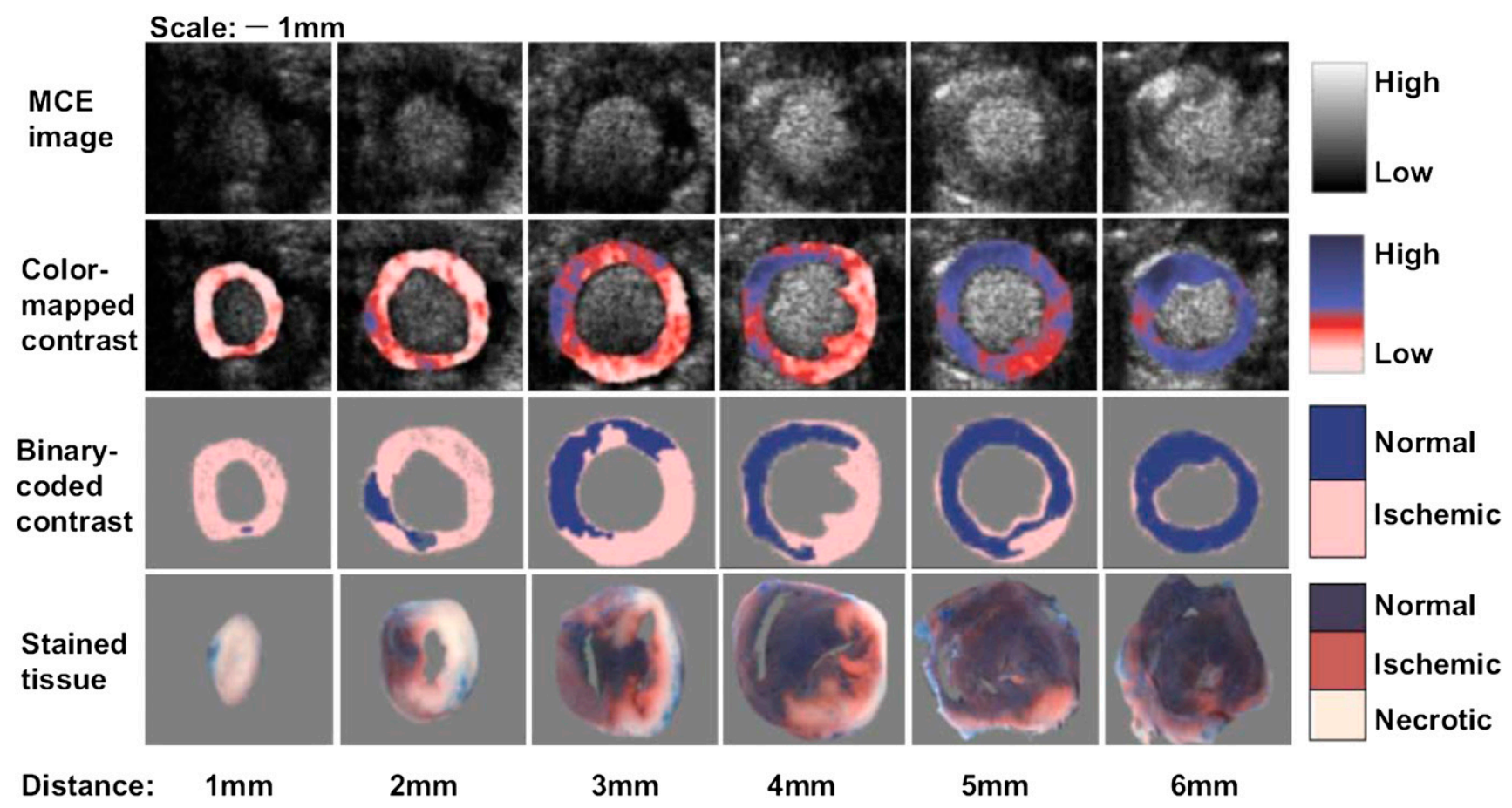

FIGURE 10. Myocardial perfusion assessed with 2-dimensional short-axis myocardial contrast echocardiography (MCE) in ischemic mouse hearts. Color-coding scheme for MCE images was selected to facilitate comparison with color photographs of triphenyltetrazolium chloride- and phthalo-blue-stained tissue sections from excised mouse hearts: normally perfused regions are in blue, ischemic regions in white to pink, and intermediately perfused regions in red. Good agreement was observed between regions identified as ischemic by MCE and those identified as ischemic or necrotic by histologic staining of postmortem tissue. (Adapted with permission of (65).)

radiotracers, ensure that PET and SPECT will continue to be key modalities in the field of molecular imaging.

Although PET and SPECT are essential modalities for molecular imaging in the mouse myocardium, it is often useful to complement the relatively low resolution and poor tissue contrast properties of PET and SPECT by coregistering these hot spot images with the high-resolution anatomic and functional images provided by CT or MRI. Although it is possible to acquire images in series and coregister them using multimodal fiducial markers, image registration is greatly facilitated using specialized instrumentation capable of applying multiple modalities in parallel (or in rapid succession within a precisely defined spatial area). In particular, the combination of PET and CT has proven as useful in preclinical imaging as it has in clinical applications. Recent developments in PET technology have now made small-animal PET/MRI a reality (72), and small-animal PET/CT and SPECT/CT systems are also commercially available (73). The impressive array of multimodal microimaging scanners currently on the market makes it fairly easy to identify an instrument that matches the expertise and facilities available within a particular institution or center.

\section{Future Challenges and Directions in Multimodality Imaging}

Although murine cardiac imaging has made impressive advances over the last decade, a great deal of work remains to be done. On the biologic side, research challenges include the development of contrast agents for molecular imaging that will provide sensitive and robust measures of thrombosis, myocardial ischemia, infarction, hibernation, angiogenesis, tissue oxygenation, $\mathrm{pH}$, redox potential, and presence of specific cell populations (neutrophils, monocyte/macrophages, fibroblasts, $\mathrm{T}$ cells, stem cells, etc.). Much progress is being made in this area, and the rapidly expanding library of available molecular imaging probes will soon make it possible to noninvasively assess nearly every key biologic process governing ischemic heart disease. On the engineering side, nearly every modality would benefit from increased sensitivity and improved temporal and spatial resolution. Although cardiac MRI is often considered the 1-stop shop capable of both functional and molecular imaging, it is not practical to keep mice under anesthesia long enough to apply every tool in that shop. By analogy, every cardiac imaging modality would benefit from further optimization to reduce the time required for image acquisition. Recent examples include parallel imaging on highfield MRI scanners and the implementation of phased-array transducers on high-frequency ultrasound systems. Another major engineering challenge lies in the development of a 1-stop shop that combines the high resolution of CT and cardiac MRI with the sensitivity of PET, SPECT, and fluorescence optical tomography. Currently, commercial systems are meeting this challenge with micro-SPECT/CT scanners 
and micro-PET/MRI scanners. However, hybrid systems using nearly every conceivable combination of imaging modality are currently being pursued in research laboratories worldwide. Although still in early development, one might predict that hybrid micro-fluorescence optical tomography/ MRI systems (74) will prove highly competitive in this regard. Novel imaging modalities, such as photoacoustic ultrasound imaging and optical coherence tomography, also show promise in specialized applications.

Although many of the advances described in this article will find application in expediting the pace of biomedical research in the laboratory, only those that are robust and provide clinically relevant endpoints will ultimately find application in clinical medicine. What is urgently needed in the diagnosis and treatment of ischemic heart disease are molecular imaging strategies that provide clinicians with information that can be used to guide clinical care or monitor the efficacy of treatment. Prime examples include the development of robust contrast agents for detecting vulnerable plaque and hibernating myocardium and for accurately measuring ischemic areas at risk in patients after reperfusion has been achieved. Significant progress toward this last goal has already been achieved using ultrasound to image ischemic memory agents, and in cardiac MRI with T2-weighted imaging, but these methods need further refinement for routine use in clinical trials.

Molecular imaging has substantial potential to shed new light on the molecular mechanisms underlying ischemic heart disease; however, this approach will find true clinical utility only in niches in which it can quickly and costeffectively change the course of medical management for individual patients. Advancing the standard of medical care is a challenging goal, particularly in managed-care environments. Ultrasound-based molecular imaging strategies may have an advantage in this regard because of the low cost and widespread availability of ultrasound scanners, followed by SPECT-, MRI-, and PET-based strategies. Another important clinical application of molecular imaging in ischemic heart disease will be in providing quantitative endpoints for use in clinical trials (e.g., quantification of hibernating or infarcted myocardium or ischemic area at risk). The accurate quantitation of endpoints such as these may not greatly affect the clinical management of individual patients, but they would nevertheless be critical in clinical trials of therapeutic agents intended to reduce infarct size by minimizing reperfusion injury.

\section{INNERVATION IMAGING AFTER MI}

Imaging Cardiac Sympathetic Nervous System

Much of the previous discussion focused on techniques that are presently applicable only in murine models of cardiac disease. We now turn to an application of molecular imaging that has made inroads into clinical use as a research and emerging clinical tool for the evaluation of patients after MI or with congestive heart failure.
It is well known that the autonomic nervous system is important for cardiac adaptation to the varying demands of daily life. It is involved in regulation of cardiac rhythm, conduction, and contractility. At the same time, the autonomic nervous system is also involved in the early response to pathologic stimuli, and sympathetic neurons seem to be more susceptible to damage than are cardiomyocytes. Thus, the autonomic nervous system constitutes an attractive target for the identification of individuals at risk of cardiovascular disease $(75,76)$. There are several radiolabeled tracers to evaluate the sympathetic nervous system that are either true catecholamines or catecholamine analogs. ${ }^{123} \mathrm{I}-\mathrm{MIBG}$ is the most commonly used SPECT tracer. It is an analog of norepinephrine that is metabolically resistant to monoamine oxidase and catechol- $O$-methyltransferase enzymes, unlike the true neurotransmitter norepinephrine. Imaging is typically performed with a 2-step protocol at 15-30 min (early phase) and 3-4 h (delayed phase) after ${ }^{123}$ I-MIBG injection, to determine uptake and washout as markers of neuronal integrity and sympathetic drive (76). In addition to ${ }^{123} \mathrm{I}-\mathrm{MIBG}$, there are several PET tracers for sympathetic nerve imaging in human studies. As it does for the assessment of perfusion, PET offers higher resolution and sensitivity for interrogation of regional tracer distribution even in situations of globally reduced uptake (i.e., heart failure), and it allows for absolute quantification from dynamic imaging data (75).

\section{Neuronal Imaging in Heart Failure}

Heart failure has become the major area of clinical interest for cardiac neuronal imaging. In heart failure, abnormalities of sympathetic innervation play a central role in initiation and progression of the disease. Merlet et al. reported for the first time the significance of ${ }^{123} \mathrm{I}-$ MIBG cardiac imaging as a predictor of outcome (77), and this was confirmed by several other studies, including the most recent prospective, multicenter ADMIRE-HF trial $(78,79)$. Decreased ${ }^{123}$ I-MIBG uptake or increased tracer washout identify high-risk patients, and these parameters seem to have an incremental prognostic value for survival above that of other clinical indices such as ejection fraction and plasma neurohormonal factors.

The pathophysiologic reasons for the value of neuronal imaging in this setting are best explained by catecholamine overexposure as a consequence of impaired neuronal reuptake into presynaptic nerve terminals $(80)$. This reuptake via the uptake-1 transporter is the most important mechanism of catecholamine clearance in the myocardium, and high tissue catecholamine levels as a consequence of impaired uptake-1 are likely to contribute to progressive loss of function.

${ }^{123} \mathrm{I}-\mathrm{MIBG}$ detects this impairment as a global myocardial phenomenon in heart failure, but presumably, alterations of innervation should occur at earlier stages, before the development of clinically overt heart failure. This impairment would be expected to be a more regional 
alteration, requiring the detailed assessment of innervation by tomographic techniques combined with concomitant perfusion assessment.

\section{Neuronal Imaging After MI}

Tomographic, integrated perfusion/innervation studies have been performed after $\mathrm{MI}$ in the experimental and clinical setting $(81,82)$. These studies suggested that cardiac sympathetic nerve terminals are more sensitive to ischemia than are myocytes (Fig. 11). In acute coronary syndromes, neuronal damage, as assessed by ${ }^{123}$ I-MIBG imaging, exceeds the area of scarring and is well correlated with the scintigraphic risk area as defined by perfusion SPECT before intervention (83). Additionally, regional abnormalities of innervation can be seen in subjects with coronary disease but without MI (84). It is believed that these areas of denervated but viable myocardium contribute to increased risk for ventricular arrhythmia. Correlations with abnormalities of repolarization and depolarization have been observed clinically (85), and an association with inducible ventricular tachycardia was recently reported in an animal model (86).

In addition to the proarrhythmic effects, impaired innervation in the viable infarct border zone is also considered to be a contributor to LV remodeling and the transition from MI to heart failure. Experimentally, a relationship between impaired ${ }^{123} \mathrm{I}-\mathrm{MIBG}$ uptake in the infarct border zone and impaired regional contractile function has been suggested (87). Additionally, some clinical studies have suggested that reduced ${ }^{123}$ I-MIBG uptake may be associated with remodeling in patients after MI (88).

\section{Future Challenges and Directions in Innervation Imaging After MI}

Although there is good and increasing evidence for the usefulness of ${ }^{123} \mathrm{I}-\mathrm{MIBG}$ in predicting outcome in heart failure, similar clinical trials for the evaluation of MI and prediction of subsequent remodeling are still missing. Characterization of the infarct border zone requires a detailed regional analysis of innervation and a comparison to regional perfusion. This is methodologically more chal- lenging than the standard planar analysis of ${ }^{123} \mathrm{I}-\mathrm{MIBG}$ uptake used in heart failure. The lack of absolute quantification with SPECT may represent a problem for regional ${ }^{123}$ I-MIBG SPECT analysis, because overall ${ }^{123}$ I-MIBG uptake may be reduced, and the usually used normalization of a SPECT study to its maximum will result in underestimation of true defect size. These issues will need to be overcome before ${ }^{123}$ I-MIBG can be translated from the global assessment of clinically overt heart failure into a regional assessment of potential precursors of heart failure. Introduction of a widely available, robust ${ }^{18} \mathrm{~F}$ labeled PET tracer of sympathetic innervation would be another approach toward establishing the quantitative regional analysis of innervation in the clinical setting. The use of PET for this purpose is currently restricted by the short half-life of ${ }^{11} \mathrm{C}$ as the label of existing established compounds.

Although myocardial innervation imaging is on its way toward clinical application in overt heart failure, it may have its greatest potential in identifying possible precursors of heart failure after ischemia and infarction. Such an application, however, is not yet well clinically established and requires further advances in methodology.

\section{CONCLUSION}

Great progress has been made in recent years in the noninvasive characterization of the molecular methods that underlie the process of LV remodeling after MI in animal models. SPECT and PET have been used to image tissue levels of MMPs, ACE, and angiotensin receptors. MRI can identify molecular processes such as apoptosis, cellular processes including macrophage infiltration, and ultrastructural changes such as collagen deposition and myofibrillar architecture. Ultrasound can also image structure and function relationships in the infarcted mouse heart and can map perfusion and reveal molecular processes with targeted imaging. However, the only methodology to date that has reached clinical fruition is neuronal imaging with ${ }^{123}$ I-MIBG. Whether the advances in molecular imaging

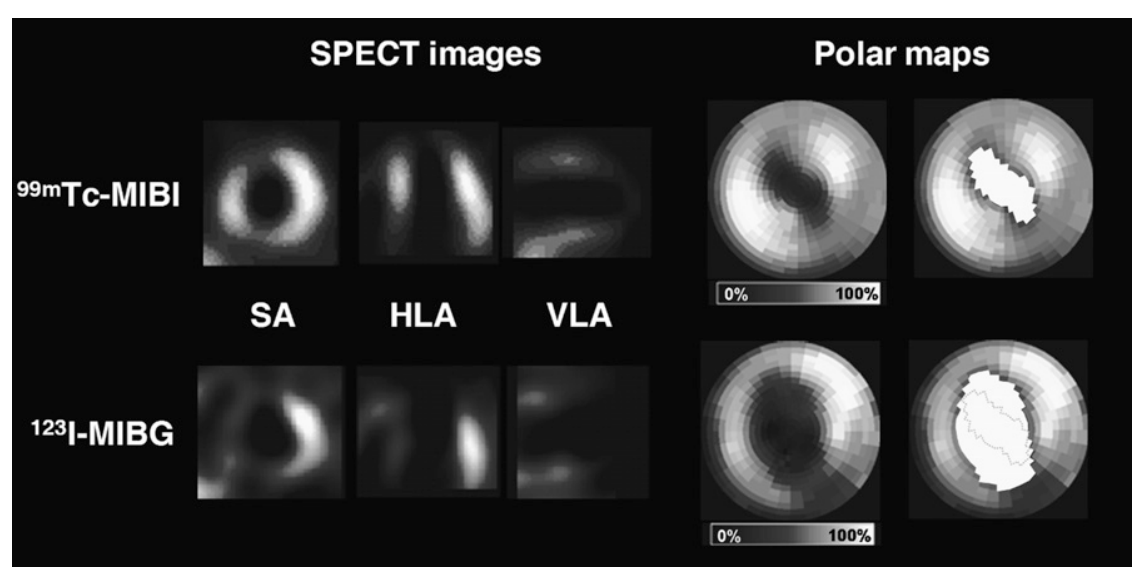

FIGURE 11. SPECT of myocardial perfusion (using 99mTc-methoxyisobutylisonitrile) and innervation (using 99mTc-MIBG) in a patient 2 wk after anteroapical MI. SPECT images and polar maps show small perfusion defect in apex and distal anteroseptal wall, along with innervation defect that exceeds perfusion defect significantly in anterior, septal, and inferior regions. Perfusion defect size (right) was calculated as $14 \%$ of polar map and innervation defect as 33\%, resulting in perfusion/innervation mismatch covering $19 \%$ of LV myocardium in infarct border zone. SA = short axis; HLA = horizontal long axis; VLA = vertical long axis. 
can be translated into improvements in patient care is the challenge for the next decade of molecular imaging.

\section{ACKNOWLEDGMENT}

This work was supported in part by grants AHA 0855409E, R01 HL078650, R01 HL065662, R01 HL093038, K08 HL079984, and R01 HL058582.

\section{REFERENCES}

1. Sutton MG, Sharpe N. Left ventricular remodeling after myocardial infarction. Pathophysiology and therapy. Circulation. 2000;101:2981-2988.

2. Weber KT. Extracellular matrix remodeling in heart failure: a role for de novo angiotensin II generation. Circulation. 1997;96:4065-4082.

3. Spinale FG. Myocardial matrix remodeling and the matrix metalloproteinases: influence on cardiac form and function. Physiol Rev. 2007;87:1285-1342.

4. Creemers EE, Davis JN, Parkhurst AM, et al. Deficiency of TIMP-1 exacerbates LV remodeling after myocardial infarction in mice. Am J Physiol Heart Circ Physiol. 2003;284:H364-H371.

5. Mukherjee R, Mingoia JT, Bruce JA, et al. Selective spatiotemporal induction of matrix metalloproteinase-2 and matrix metalloproteinase-9 transcription following myocardial infarction. Am J Physiol Heart Circ Physiol. 2006;291:22162228.

6. Wilson EM, Moainie SL, Baskin JM, et al. Region- and type-specific induction of matrix metalloproteinases in post-myocardial infarction remodeling. Circulation. 2003; 107:2857-2863.

7. Webb CS, Bonnema DD, Ahmed SH, et al. Specific temporal profile of matrix metalloproteinase release occurs in patients after myocardial infarction: relation to left ventricular remodeling. Circulation. 2006;114:1020-1027.

8. Chen J, Tung CH, Allport JR, Chen S, Weissleder R, Huang PL. Near-infrared fluorescent imaging of matrix metalloproteinase activity after myocardial infarction. Circulation. 2005;111:1800-1805.

9. Su H, Spinale FG, Dobrucki LW, et al. Noninvasive targeted imaging of matrix metalloproteinase activation in a murine model of postinfarction remodeling. Circulation. 2005;112:3157-3167.

10. Sahul Z, Song J, McAteer J, et al. Quantification of in-vivo matrix metalloproteinase activity and myocardial strain yield unique spatial temporal patterns in a porcine model of myocardial infarction [abstract]. Circulation. 2006;114:II_500.

11. Li S, Dobrucki LW, Sinusas AJ, Liu YH. A new method for SPECT quantification of targeted radiotracers uptake in the myocardium. Med Image Comput Comput Assist Interv. 2005;8:684-691.

12. Liu Y, Sahul Z, Weyman C, et al. Hotspot quantification of myocardial focal tracer uptake from molecular targeted SPECT/CT images: canine validation. Proc SPIE. 2008;6915:69150N1-8.

13. Paul M, Poyan Mehr A, Kreutz R. Physiology of local renin-angiotensin systems. Physiol Rev. 2006;86:747-803

14. Shirani J, Dilsizian V. Imaging left ventricular remodeling: targeting the neurohumoral axis. Nat Clin Pract Cardiovasc Med. 2008;5(suppl 2):S57-S62.

15. Dilsizian V, Eckelman WC, Loredo ML, Jagoda EM, Shirani J. Evidence for tissue angiotensin-converting enzyme in explanted hearts of ischemic cardiomyopathy using targeted radiotracer technique. J Nucl Med. 2007;48: $182-187$.

16. Shirani J, Narula J, Eckelman WC, Narula N, Dilsizian V. Early imaging in heart failure: exploring novel molecular targets. J Nucl Cardiol. 2007;14:100-110.

17. Verjans JW, Lovhaug D, Narula N, et al. Noninvasive imaging of angiotensin receptors after myocardial infarction. JACC Cardiovasc Imaging. 2008;1:354-362.

18. Streeter DD Jr, Hanna WT. Engineering mechanics for successive states in canine left ventricular myocardium. II. Fiber angle and sarcomere length. Circ Res. 1973;33:656-664.

19. Streeter DD Jr, Spotnitz HM, Patel DP, Ross J Jr, Sonnenblick EH. Fiber orientation in the canine left ventricle during diastole and systole. Circ Res. 1969;24:339-347.

20. Buckberg G, Hoffman JI, Mahajan A, Saleh S, Coghlan C. Cardiac mechanics revisited: the relationship of cardiac architecture to ventricular function. Circulation. 2008;118:2571-2587.

21. Kramer CM. Insights into myocardial microstructure during infarct healing and remodeling: pathologists need not apply. Circ Cardiovasc Imaging. 2009;2:4-5.

22. Hagmann P, Jonasson L, Maeder P, Thiran JP, Wedeen VJ, Meuli R. Understanding diffusion MR imaging techniques: from scalar diffusion-weighted imaging to diffusion tensor imaging and beyond. Radiographics. 2006;26(suppl 1):S205-S223.

23. Sosnovik DE, Wang R, Dai G, et al. Diffusion spectrum MRI tractography reveals the presence of a complex network of residual myofibers in infarcted myocardium. Circ Cardiovasc Imaging. 2009;2:206-212.

24. Wedeen VJ, Hagmann P, Tseng WY, Reese TG, Weisskoff RM. Mapping complex tissue architecture with diffusion spectrum magnetic resonance imaging. Magn Reson Med. 2005;54:1377-1386.

25. Sosnovik DE, Wang R, Dai G, Reese TG, Wedeen VJ. Diffusion MR tractography of the heart. J Cardiovasc Magn Reson. 2009;11:47.

26. Sosnovik DE, Nahrendorf M, Weissleder R. Molecular magnetic resonance imaging in cardiovascular medicine. Circulation. 2007;115:2076-2086.

27. Caravan P, Das B, Dumas S, et al. Collagen-targeted MRI contrast agent for molecular imaging of fibrosis. Angew Chem Int Ed Engl. 2007;46:8171-8173.

28. Spuentrup E, Ruhl KM, Botnar RM, et al. Molecular magnetic resonance imaging of myocardial perfusion with EP-3600, a collagen-specific contrast agent: initial feasibility study in a swine model. Circulation. 2009;119:1768-1775.

29. Helm PA, Caravan P, French BA, et al. Postinfarction myocardial scarring in mice: molecular MR imaging with use of a collagen-targeting contrast agent. Radiology. 2008;247:788-796.

30. Sosnovik DE, Nahrendorf M, Weissleder R. Magnetic nanoparticles for MR imaging: agents, techniques and cardiovascular applications. Basic Res Cardiol. 2008; 103:122-130.

31. Krombach GA, Wendland MF, Higgins CB, Saeed M. MR imaging of spatial extent of microvascular injury in reperfused ischemically injured rat myocardium: value of blood pool ultrasmall superparamagnetic particles of iron oxide. Radiology. 2002;225:479-486.

32. Sosnovik DE, Garanger E, Aikawa E, et al. Molecular MRI of cardiomyocyte apoptosis with simultaneous delayed-enhancement MRI distinguishes apoptotic and necrotic myocytes in vivo: potential for midmyocardial salvage in acute ischemia. Circ Cardiovasc Imaging. 2009;2:460-467.

33. Harisinghani MG, Barentsz J, Hahn PF, et al. Noninvasive detection of clinically occult lymph-node metastases in prostate cancer. $N$ Engl J Med. 2003;348:24912499.

34. Sosnovik DE, Nahrendorf M, Panizzi P, et al. Molecular MRI detects low levels of cardiomyocyte apoptosis in a transgenic model of chronic heart failure. Circ Cardiovasc Imaging. 2009;2:468-475.

35. Montet X, Montet-Abou K, Reynolds F, Weissleder R, Josephson L. Nanoparticle imaging of integrins on tumor cells. Neoplasia. 2006;8:214-222.

36. Taktak S, Sosnovik D, Cima MJ, Weissleder R, Josephson L. Multiparameter magnetic relaxation switch assays. Anal Chem. 2007;79:8863-8869.

37. Nahrendorf M, Swirski FK, Aikawa E, et al. The healing myocardium sequentially mobilizes two monocyte subsets with divergent and complementary functions. J Exp Med. 2007;204:3037-3047.

38. Farrar CT, Dai G, Novikov M, et al. Impact of field strength and iron oxide nanoparticle concentration on the linearity and diagnostic accuracy of offresonance imaging. NMR Biomed. 2008;21:453-463.

39. Sosnovik DE, Nahrendorf M, Deliolanis N, et al. Fluorescence tomography and magnetic resonance imaging of myocardial macrophage infiltration in infarcted myocardium in vivo. Circulation. 2007;115:1384-1391.

40. Sosnovik DE, Schellenberger EA, Nahrendorf M, et al. Magnetic resonance imaging of cardiomyocyte apoptosis with a novel magneto-optical nanoparticle. Magn Reson Med. 2005;54:718-724.

41. Chen JW, Querol Sans M, Bogdanov A Jr, Weissleder R. Imaging of myeloperoxidase in mice by using novel amplifiable paramagnetic substrates. Radiology. 2006;240:473-481.

42. Nahrendorf M, Sosnovik D, Chen JW, et al. Activatable magnetic resonance imaging agent reports myeloperoxidase activity in healing infarcts and noninvasively detects the antiinflammatory effects of atorvastatin on ischemiareperfusion injury. Circulation. 2008;117:1153-1160.

43. Weissleder R, Ntziachristos V. Shedding light onto live molecular targets. Nat Med. 2003;9:123-128.

44. Mahmood U, Tung CH, Bogdanov A Jr, Weissleder R. Near-infrared optical imaging of protease activity for tumor detection. Radiology. 1999;213:866870 .

45. Graves EE, Ripoll J, Weissleder R, Ntziachristos V. A submillimeter resolution fluorescence molecular imaging system for small animal imaging. Med Phys. 2003;30:901-911.

46. Deliolanis N, Lasser T, Hyde D, Soubret A, Ripoll J, Ntziachristos V. Free-space fluorescence molecular tomography utilizing 360 degrees geometry projections. Opt Lett. 2007;32:382-384.

47. Nahrendorf M, Sosnovik DE, Waterman P, et al. Dual channel optical tomographic imaging of leukocyte recruitment and protease activity in the healing myocardial infarct. Circ Res. 2007;100:1218-1225. 
48. Vinegoni C, Razansky D, Figueiredo JL, et al. Born normalization for fluorescence optical projection tomography for whole heart imaging. Available at: http://www.jove.com/index/Details.stp?ID=1389. Accessed February 24, 2010.

49. Vinegoni C, Razansky D, Figueiredo JL, Nahrendorf M, Ntziachristos V, Weissleder R. Normalized Born ratio for fluorescence optical projection tomography. Opt Lett. 2009;34:319-321.

50. Yang Z, Berr SS, Gilson WD, Toufektsian M-C, French BA. Simultaneous evaluation of infarct size and cardiac function in intact mice by contrastenhanced cardiac magnetic resonance imaging reveals contractile dysfunction in noninfarcted regions early after myocardial infarction. Circulation. 2004;109: 1161-1167.

51. Ross AJ, Yang Z, Berr S, et al. Serial MRI evaluation of cardiac structure and function in mice after reperfused myocardial infarction. Magn Reson Med. 2002;47:1158-1168.

52. Gilson WD, Yang Z, French BA, Epstein FH. Measurement of myocardial mechanics in mice before and after infarction using multislice displacementencoded MRI with 3D motion encoding. Am J Physiol Heart Circ Physiol. 2005;288:H1491-H1497.

53. Young AA, French BA, Yang Z, et al. Reperfused myocardial infarction in mice: 3D mapping of late gadolinium enhancement and strain. J Cardiovasc Magn Reson. 2006;8:685-692.

54. Chapon C, Herlihy A, Bhakoo K. Assessment of myocardial infarction in mice by late gadolinium enhancement MR imaging using an inversion recovery pulse sequence at 9.4T. J Cardiovasc Magn Reson. 2008;10:6.

55. Beyers R, Xu Y, Epstein F, French B. T2-weighted MRI pulse sequences for imaging post-infarct edema in mice: comparison of spin echo and $\mathrm{T} 2$ preparation approaches [abstract]. J Cardiovasc Magn Reson. 2009;11(suppl 1):O33.

56. Streif JUG, Nahrendorf M, Hiller K-H, et al. In vivo assessment of absolute perfusion and intracapillary blood volume in the murine myocardium by spin labeling magnetic resonance imaging. Magn Reson Med. 2005;53:584-592.

57. Kramer CM. Integrated approach to ischemic heart disease: the one-stop shop. Cardiol Clin. 1998;16:267-276.

58. Gilson WD, Epstein FH, Yang Z, et al. Borderzone contractile dysfunction is transiently attenuated and left ventricular structural remodeling is markedly reduced following reperfused myocardial infarction in inducible nitric oxide synthase knockout mice. J Am Coll Cardiol. 2007;50:1799-1807.

59. Nahrendorf M, Streif JU, Hiller K-H, et al. Multimodal functional cardiac MRI in creatine kinase-deficient mice reveals subtle abnormalities in myocardial perfusion and mechanics. Am J Physiol Heart Circ Physiol. 2006;290:H25162521.

60. Timmers L, Sluijter JPG, van Keulen JK, et al. Toll-like receptor 4 mediates maladaptive left ventricular remodeling and impairs cardiac function after myocardial infarction. Circ Res. 2008;102:257-264.

61. Yang Z, Bove CM, French BA, et al. Angiotensin II type 2 receptor overexpression preserves left ventricular function after myocardial infarction. Circulation. 2002;106:106-111.

62. Foster FS, Charles JP, Kasia AH, Donald AC, Daniel HT. Advances in ultrasound biomicroscopy. Ultrasound Med Biol. 2000;26:1-27.

63. Dawson D, Lygate CA, Saunders J, et al. Quantitative 3-dimensional echocardiography for accurate and rapid cardiac phenotype characterization in mice. Circulation. 2004;110:1632-1637.

64. Li Y, Garson CD, Xu Y, et al. Quantification and MRI validation of regional contractile dysfunction in mice post myocardial infarction using high resolution ultrasound. Ultrasound Med Biol. 2007;33:894-904.

65. French BA, Li Y, Klibanov AL, Yang Z, Hossack JA. 3D perfusion mapping in post-infarct mice using myocardial contrast echocardiography. Ultrasound Med Biol. 2006;32:805-815.

66. Ebert SN, Taylor DG, Nguyen H-L, et al. Noninvasive tracking of cardiac embryonic stem cells in vivo using magnetic resonance imaging techniques. Stem Cells. 2007;25:2936-2944.

67. Lindner JR, Song J, Xu F, et al. Noninvasive ultrasound imaging of inflammation using microbubbles targeted to activated leukocytes. Circulation. 2000;102: 2745-2750.
68. Villanueva FS, Lu E, Bowry S, et al. Myocardial ischemic memory imaging with molecular echocardiography. Circulation. 2007;115:345-352.

69. Constantinesco A, Choquet P, Monassier L, Israel-Jost V, Mertz L. Assessment of left ventricular perfusion, volumes, and motion in mice using pinhole gated SPECT. J Nucl Med. 2005;46:1005-1011.

70. Yang Y, Rendig S, Siegel S, Newport DF, Cherry SR. Cardiac PET imaging in mice with simultaneous cardiac and respiratory gating. Phys Med Biol. 2005; 50:2979-2989.

71. Thomas D, Bal H, Arkles J, et al. Noninvasive assessment of myocardial viability in a small animal model: comparison of MRI, SPECT, and PET. Magn Reson Med. 2008;59:252-259.

72. Judenhofer MS, Catana C, Swann BK, et al. PET/MR images acquired with a compact MR-compatible PET detector in a 7-T magnet. Radiology. 2007; 244:807-814.

73. Del Guerra A, Belcari N. State-of-the-art of PET, SPECT and CT for small animal imaging. Nucl Instrum Methods Phys Res A. 2007;583:119-124.

74. Davis S, Pogue B, Springett R, et al. Magnetic resonance-coupled fluorescence tomography scanner for molecular imaging of tissue [abstract]. Rev Sci Instrum. 2008;79:064302.

75. Bengel FM, Schwaiger M. Assessment of cardiac sympathetic neuronal function using PET imaging. J Nucl Cardiol. 2004;11:603-616.

76. Carrio I. Cardiac neurotransmission imaging. J Nucl Med. 2001;42:1062-1076.

77. Merlet P, Valette H, Dubois-Rande JL, et al. Prognostic value of cardiac metaiodobenzylguanidine imaging in patients with heart failure. J Nucl Med. 1992;33:471-477.

78. Agostini D, Verberne HJ, Burchert W, et al. I-123-MIBG myocardial imaging for assessment of risk for a major cardiac event in heart failure patients: insights from a retrospective European multicenter study. Eur J Nucl Med Mol Imaging. 2008:35:535-546.

79. Jacobson AF, Lombard J, Banerjee G, Camici PG. ${ }^{123}$ I-MIBG scintigraphy to predict risk for adverse cardiac outcomes in heart failure patients: design of two prospective multicenter international trials. J Nucl Cardiol. 2009;16:113121.

80. Esler M, Jennings G, Lambert G, Meredith I, Horne M, Eisenhofer G. Overflow of catecholamine neurotransmitters to the circulation: source, fate, and functions. Physiol Rev. 1990;70:963-985.

81. Dae MW, Herre JM, O'Connell JW, Botvinick EH, Newman D, Munoz L. Scintigraphic assessment of sympathetic innervation after transmural versus nontransmural myocardial infarction. J Am Coll Cardiol. 1991;17:14161423 .

82. McGhie AI, Corbett JR, Akers MS, et al. Regional cardiac adrenergic function using I-123 meta-iodobenzylguanidine tomographic imaging after acute myocardial infarction. Am J Cardiol. 1991;67:236-242.

83. Matsunari I, Schricke U, Bengel FM, et al. Extent of cardiac sympathetic neuronal damage is determined by the area of ischemia in patients with acute coronary syndromes. Circulation. 2000;101:2579-2585.

84. Bulow HP, Stahl F, Lauer B, et al. Alterations of myocardial presynaptic sympathetic innervation in patients with multi-vessel coronary artery disease but without history of myocardial infarction. Nucl Med Commun. 2003;24: 233-239.

85. Simoes MV, Barthel P, Matsunari I, et al. Presence of sympathetically denervated but viable myocardium and its electrophysiologic correlates after early revascularised, acute myocardial infarction. Eur Heart J. 2004;25:551557.

86. Sasano T, Abraham MR, Chang KC, et al. Abnormal sympathetic innervation of viable myocardium and the substrate of ventricular tachycardia after myocardial infarction. J Am Coll Cardiol. 2008;51:2266-2275.

87. Kramer CM, Nicol PD, Rogers WJ, et al. Reduced sympathetic innervation underlies adjacent noninfarcted region dysfunction during left ventricular remodeling. J Am Coll Cardiol. 1997;30:1079-1085.

88. Sakata K, Mochizuki M, Yoshida H, et al. Cardiac sympathetic dysfunction contributes to left ventricular remodeling after acute myocardial infarction. Eur J Nucl Med. 2000;27:1641-1649. 